\title{
A delay equation model for oviposition habitat selection by mosquitoes
}

\author{
Stephen A. Gourley ${ }^{a, *}$ and Shigui Ruan ${ }^{b, \dagger}$ \\ ${ }^{a}$ Department of Mathematics, University of Surrey, \\ Guildford, Surrey, GU2 7XH, UK. \\ ${ }^{b}$ Department of Mathematics, University of Miami, \\ Coral Gables, FL 33124-4250, USA.
}

September 19, 2011

\begin{abstract}
We propose a patch type model for mosquitoes that have aquatic larvae inhabiting ponds. Partial differential equations (PDEs) model the larvae on each of several disconnected patches representing the ponds, with conditions varying in each patch, coupled via the adults in the air. From the PDEs a scalar delay differential equation, with multiple delays, for the total adult mosquito population is derived. The various delays represent the larval development times in the patches. The coefficients contain all the relevant information about the sizes and geometry of the individual patches inhabited by the larvae, the boundary conditions applicable to those patches and the diffusivity of the larvae in each patch. For patches of general shapes and sizes, and without the need to specify the criteria by which an adult mosquito selects an oviposition patch, the modern theory of monotone dynamical systems and persistence theory enables a complete determination of the conditions for the mosquito population to go extinct or to persist. More detailed biological insights are obtained for the case when the patches are squares of various sizes, which allows a detailed discussion of the effects of scale, and for two particular criteria by which mosquitoes might select patches for oviposition, being (i) selection based solely on patch area, and (ii) selection based both on area and expected larval survival probability for each patch. In some parameter regimes, counterintuitive phenomena are predicted.
\end{abstract}

Keywords: Oviposition, mosquito, delay, patch, persistence.

Mathematics Subject Classification: 34K20, 35K57, 92D40.

*Corresponding author. E-mail: s.gourley@surrey.ac.uk. Fax: +44 1483686071

${ }^{\dagger}$ Research was partially supported by National Science Foundation grant DMS-1022728. 


\section{Introduction}

Ovipositing insects, in particular species in which juveniles cannot disperse away from low quality habitats, often select sites that improve the survival, growth and reproduction of their offspring (Bond et al. [7]). It has been demonstrated that mosquitoes select oviposition habitats in response to physical and chemical suitability for larval development (Bentley and Day [3]), habitat size (Heard [13]), larval food resource levels (Blaustein and Kotler [5]), the presence of competitors (Edgerly et al. [6]), and the presence of predators (Blaustein et al. [4], Eitam and Blaustein [9], Spencer et al. [18]). Since applying larvicides to acquatic habitats is one of the most effective means of controlling mosquito-borne diseases (WHO [20]), recently there have been some very interesting studies on reduction of mosquito larval habitats through environmental management ( $\mathrm{Gu}$ et al. [10]). We refer to $\mathrm{Gu}$ et al. [11] for more discussions and references on this subject.

In this paper we develop and study a patch type model for insects such as mosquitoes that have aquatic larvae that live in ponds. An important issue that arises in this and many other ecological scenarios is that adults and juveniles have completely different characteristics and view their spatial environment in completely different ways. This is an important point because in interacting species models in ecology it is usually assumed that there is just one spatial domain $\Omega$ in which all species, or perhaps the individuals of all life stages in a particular species, reside. However, in reality different species often perceive space in different ways and also at different scales — this is especially true of airborne predators feeding on prey that are confined to patches with boundaries. Even when modelling a single species interacting only with itself, individuals may perceive space differently at different stages of life. Larval mosquitoes live in ponds. But when a larva matures into an adult, it takes to the air and may lay its eggs in another pond. Using the modelling methodology we present in this paper, this leads to partial differential equations for the mosquito larvae on each of several disconnected domains $\Omega_{i}, i=1,2, \ldots, n$, with conditions varying in each domain, coupled via the adults in the air. Here the adults are not subject to the boundary conditions that apply to the larvae in each $\Omega_{i}$, but instead view space as one single, much larger, domain of which each $\Omega_{i}$ is a subset.

Our modelling methodology allows us to derive a single autonomous delay differential equation, with multiple delays, for the total adult mosquito population. The various delays are the larval development times in the various ponds. The coefficients of this scalar delay equation contain all the relevant information about the sizes and geometry of the individual ponds inhabited by the larvae, the boundary conditions applicable to those ponds and the diffusivity of the larvae in each pond. The availability of such a model enables us to apply the modern theory of monotone dynamical systems and persistence theory to determine completely the conditions for the mosquito population to go extinct or persist. Moreover, this can be done in a rather general setting where the criteria by which adult mosquitoes select ponds for oviposition are not specified in detail. 
Later we specialise to the case where the ponds, or the mathematical patches which represent them in a sense described below, are squares of various sizes. This enables a certain Green's function which arises in the model derivation to be explicitly computed which later facilitates a detailed study of the role of the sizes of the ponds. We also investigate in detail two particular possible strategies by which mosquitoes might select oviposition ponds. These are (i) pond selection based only on area, whereby larger ponds are more likely to be chosen for oviposition and mosquitoes apply no other criteria, and (ii) pond selection based both on area and larval survival probability in each pond. The latter is an attempt to model the fact that in nature several taxa, including mosquitoes, tend to avoid oviposition in ponds known to contain predators of larvae (Spencer et al. [18]). For example, the larvae of the mosquito Culiseta longiareolata are vulnerable to predation by the backswimmer Nononecta maculata and adult mosquitoes can detect the presence of this predator in a pond at very low densities.

Our model allows the boundary conditions to be either of homogeneous Dirichlet or homogeneous Neumann type, or a mixture of the two (i.e. different boundary conditions in different ponds). One might suppose that the only correct boundary conditions to apply to larvae in ponds would be homogeneous Neumann conditions (i.e. no flux conditions), since larvae are physically confined to ponds. This seems reasonable in the case of small ponds containing no predators, where the water is calm everywhere and larvae can be expected to be found in all parts of the pond. However, for larger ponds the reality is not so simple. Larvae inhabiting larger ponds tend to be found only in the shallower, calmer, protected water near the edge of the pond, which may be inaccessible to fish and other predators that might prey on them. One therefore anticipates that the larvae density will drop off sharply with increasing depth near the centre of the pond, and therefore that for a particular pond the effective larval habitat is really an annular (not necessarily circular) subset of the pond near the boundary. It would then be reasonable to apply homogeneous Neumann boundary conditions at the outer boundary (the true edge of the pond), and homogeneous Dirichlet boundary conditions at the inner boundary which represents the far more hostile interior of the pond. Larvae could, but should not, cross the inner boundary. Now, such problems are in some cases mathematically equivalent to other problems on larger domains where homogeneous Dirichlet conditions are applied on all parts of the boundary. For example, solutions of the scalar problem $u_{t}=D u_{x x}+f(u), x \in(0, l)$, subject to $u_{x}(0, t)=0$, $u(l, t)=0$ are symmetric solutions of the Dirichlet problem $u_{t}=D u_{x x}+f(u)$, $x \in(-l, l), u( \pm l, t)=0$. The latter problem is on a larger domain $(-l, l)$ the size of which scales with $l$ in the same way as the "true" domain $(0, l)$. The same is true in this paper and we will adopt a distinction between the words pond and patch. The pond is the true biological larval habitat. There is no distinction between patch and pond where we are considering small ponds with homogeneous Neumann boundary conditions at the edge, and no inner boundary. Where we are considering a larger pond with an inhabitable zone only near the edge, an inner boundary bordering the uninhabitable predator-infested interior, and Neumann 
and Dirichlet boundary conditions applying at these respective boundaries, we shall assume that the pond can be "represented" by some other spatial region that we call a patch, which scales in area with the inhabitable zone of the pond but has only an outer boundary on which homogeneous Dirichlet boundary conditions apply. This seems reasonable since the effects of scale are important in this paper. When we talk about a patch representing a pond, this is what we mean. Where there is a distinction between the two, the notation $\Omega_{i}$ refers to the patch rather than the pond.

A fair amount of emphasis is given to the case of homogeneous Dirichlet boundary conditions on all patches. The reader should understand that in this case the patches are not the actual larval habitats (the edges of ponds) but simply indirect representations of those habitats. For the actual ponds, Neumann boundary conditions apply on their outer boundaries, and Dirichlet conditions on the inner boundaries. For the case of square patches of various sizes with homogeneous Dirichlet boundary conditions, if the patches are all scaled down in size by the same scaling factor then extinction is the outcome once they are downsized sufficiently. A similar outcome holds for a simple KISS type scalar reaction diffusion equation on a finite domain under homogeneous Dirichlet boundary conditions. However, our model sometimes predicts maximum as well as minimum patch (and therefore also pond) sizes for survival. Moreover, if one particular patch is increased in size leaving others unchanged, it is possible under certain circumstances that extinction can be the outcome for the entire mosquito population, not just for larvae in the pond represented by that particular patch. If conditions in the pond being enlarged are such that larvae have poor survival prospects in that pond then, although enlarging the pond attracts more egg-laying adults, it is a bad pond for egg-laying and it is possible for the entire population of adults, and the larvae in all ponds, to be eradicated.

A particularly novel outcome of the analysis is that for two ponds it is possible for mosquitoes to survive when they base their oviposition pond selection on area only, but go extinct if they also try to take account of the likelihood of their larvae surviving to maturation in each pond. This is not the first time that this counterintuitive outcome has been predicted, but we emphasize that it only happens if the per-capita adult death rate at low densities is small and both ponds have hostile interiors with small habitable zones. It seems likely that the parameter values necessary for this particular outcome are unrealistic.

\section{Model derivation}

We assume there are $n$ distinct non-overlapping patches $\Omega_{i}, i=1,2, \ldots, n$. In the sense just described, these represent the habitable zones around the edges of ponds of water, or the whole pond if it is small and all parts of it are larvae-friendly,

and they are not restricted in size or shape at this stage. Juvenile mosquitoes are larvae, each of which spends its juvenile stage in a particular pond and can only 
move within that pond and not to other ponds or into the air. To derive a model, we let $A(t)$ denote the total number of adult insects, all of which are assumed to be in the air. This is perhaps not strictly accurate since an adult has to associate itself with a particular pond for egg laying even if the visit to that pond is brief. As an approximation we assume that adults drop eggs into ponds from the air, making no contact with the water surface. For some genera this is exactly what happens (see Bentley and Day [3]). Pond selection by adults can be driven by a number of factors, an important one of which is pond size as measured here by area. The importance of pond area is indicated by experimental work using the mosquito Culiseta longiareolata which suggests that the number of egg rafts laid will increase roughly linearly with pond surface area (Blaustein et al. [4]). Thus, we denote $\left|\Omega_{i}\right|$ to be the area of pond $i$ (or the patch which represents it) and $|\Omega|$ to be the total area so that, since the patches are non-overlapping, $|\Omega|=\sum_{i=1}^{n}\left|\Omega_{i}\right|$. A juvenile (larval) insect will always be in a particular pond, so we let $J_{i}(t, \mathbf{x})$ be the density of larvae at time $t$ in pond $i$. Although not always mathematically necessary it is sensible to think of space as two-dimensional because larvae breath atmospheric air and therefore stay close to the water surface for air exchange. Thus, density is thought of as the number of larvae per unit area.

Following a well developed age-structured modelling approach, for patch/pond $i$ we let $u_{i}(t, a, \mathbf{x})$ be the density of juveniles of age $a$ at point $\mathbf{x} \in \Omega_{i}$ at time $t$. For simplicity we assume that juveniles in pond $i$ diffuse within that pond according to Fick's law of diffusion with diffusivity $d_{i}$ and experience a constant per-capita mortality of $\mu_{i}$. The latter is very much a simplifying assumption because younger larvae may be subject to cannibalism by older larvae (laboratory experiments by Koenraadt and Takken [14]), or resource depletion or pollution of their environment by conspecifics (Bédhomme et al. [2]), which may also increase developmental time. Intra-specific competition in food-limited habitats can also be a factor (Barrera [1]). Any such factors clearly influence the maturation rate and will complicate the details of the mathematical formulation where they imply that $\mu_{i}$ is not really a constant. We assume that a juvenile in pond $i$ remains a juvenile until reaching the maturation age $\tau_{i}$ for that pond when it becomes an adult and leaves the pond for the air. For the evolution in pond $i$, we have

$$
\left(\frac{\partial}{\partial t}+\frac{\partial}{\partial a}\right) u_{i}(t, a, \mathbf{x})=d_{i} \nabla^{2} u_{i}(t, a, \mathbf{x})-\mu_{i} u_{i}(t, a, \mathbf{x}) \quad \text { in } \Omega_{i}
$$

under some boundary and initial conditions to be discussed further below. Let

$$
u_{i}^{\xi}(a, \mathbf{x})=u_{i}(a+\xi, a, \mathbf{x}) .
$$

Then

$$
\frac{\partial u_{i}^{\xi}(a, \mathbf{x})}{\partial a}=d_{i} \nabla^{2} u_{i}^{\xi}(a, \mathbf{x})-\mu_{i} u_{i}^{\xi}(a, \mathbf{x}) .
$$

The solution of this is

$$
u_{i}^{\xi}(a, \mathbf{x})=e^{-\mu_{i} a} \int_{\Omega_{i}} G_{i}(a, \mathbf{x}, \mathbf{y}) u_{i}^{\xi}(0, \mathbf{y}) d \mathbf{y}
$$


where $G_{i}(a, \mathbf{x}, \mathbf{y})$ is the Green's function satisfying

$$
\frac{\partial G_{i}}{\partial a}=d_{i} \nabla_{\mathbf{x}}^{2} G_{i} \quad \text { in } \Omega_{i}, \quad G_{i}(0, \mathbf{x}, \mathbf{y})=\delta(\mathbf{x}-\mathbf{y})
$$

and whatever spatial boundary conditions are to be applied to (2.1) itself. In (2.3), $\nabla_{\mathbf{x}}^{2}$ means the Laplacian calculated with respect to the first spatial argument $\mathbf{x} \in \Omega_{i}$ of $G_{i}(a, \mathbf{x}, \mathbf{y})$, treating $\mathbf{y}$ as a constant vector. It is possible to include homogeneous Neumann or homogeneous Dirichlet boundary conditions as particular cases. We shall consider these cases later.

Setting $\xi=t-\tau_{i}$ and $a=\tau_{i}$ in $(2.2)$ gives

$$
u_{i}\left(t, \tau_{i}, \mathbf{x}\right)=e^{-\mu_{i} \tau_{i}} \int_{\Omega_{i}} G_{i}\left(\tau_{i}, \mathbf{x}, \mathbf{y}\right) u_{i}\left(t-\tau_{i}, 0, \mathbf{y}\right) d \mathbf{y}
$$

which is the maturation rate per unit area at location $\mathbf{x} \in \Omega_{i}$. The maturation rate involves the birth rate $u_{i}\left(t-\tau_{i}, 0, \mathbf{y}\right)$ per unit area at location $\mathbf{y}$ at the time $t-\tau_{i}$ when the individuals maturing at time $t$ were born. An individual which matures at point $\mathbf{x} \in \Omega_{i}$ could have been born at another point $\mathbf{y} \in \Omega_{i}$ and then moved to $\mathbf{x}$ to mature. The integral totals up the contributions from all possible birth locations, the Green's function can be thought of as a probability distribution function which describes the likelihood of moving from $\mathbf{y}$ to $\mathbf{x}$, and the exponential factor $e^{-\mu_{i} \tau_{i}}$ is the probability of survival to maturation age $\tau_{i}$. In the case of the homogeneous Neumann problem, with $\nabla u_{i} \cdot \mathbf{n}=0$ on $\partial \Omega_{i}$, where $\mathbf{n}$ is the outward pointing unit normal to $\partial \Omega_{i}$,

$$
\int_{\Omega_{i}} G_{i}(a, \mathbf{x}, \mathbf{y}) d \mathbf{x}=\int_{\Omega_{i}} G_{i}(a, \mathbf{x}, \mathbf{y}) d \mathbf{y}=1 \quad \text { for all } a \geq 0 .
$$

However, this is not so for the case of the homogeneous Dirichlet problem when $u_{i}=0$ on $\partial \Omega_{i}$. In this case a larva could die by entering the lethal predatorinfested interior of the pond, which corresponds to the boundary $\partial \Omega_{i}$ of the patch representing the pond. In this case the Green's function $G_{i}(a, \mathbf{x}, \mathbf{y})$ is different and no longer integrates to 1 for all $a>0$.

Since juveniles in pond $i$ constitute those individuals in the pond which have age no greater than $\tau_{i}$,

$$
J_{i}(t, \mathbf{x})=\int_{0}^{\tau_{i}} u_{i}(t, a, \mathbf{x}) d a
$$

Differentiating this with respect to $t$ and using (2.1) gives

$$
\frac{\partial J_{i}(t, \mathbf{x})}{\partial t}=d_{i} \nabla^{2} J_{i}(t, \mathbf{x})+u_{i}(t, 0, \mathbf{x})-u_{i}\left(t, \tau_{i}, \mathbf{x}\right)-\mu_{i} J_{i}(t, \mathbf{x}) .
$$

The terms $u_{i}(t, 0, \mathbf{x})$ and $u_{i}\left(t, \tau_{i}, \mathbf{x}\right)$ are, respectively, the birth rate and maturation rate per unit area at $\mathbf{x} \in \Omega_{i}$. The latter is given by (2.4) and, as just noted, 
involves the birth rate at an earlier time. It is now necessary to decide on an appropriate expression for the birth rate. We argue that the overall egg laying rate (not the rate per unit area) at a particular pond $\Omega_{i}$ is a function $b_{i}$ of the number of adults likely to choose that particular pond, for whatever reason. So, let $p_{i}$ be the probability that an adult insect will choose pond $i$ to lay its eggs. Then $\sum_{i=1}^{n} p_{i}=1$ and the number of adults available for egg laying at pond $i$ is $p_{i} A(t)$. The actual rate of egg laying at that pond is then $b_{i}\left(p_{i} A(t)\right)$. However we need the rate per unit area so we divide by $\left|\Omega_{i}\right|$. This gives

$$
u_{i}(t, 0, \mathbf{x})=\frac{1}{\left|\Omega_{i}\right|} b_{i}\left(p_{i} A(t)\right)
$$

which is actually independent of $\mathbf{x}$.

There are various possible ways to choose the $p_{i}$. One possibility is to assume that the probability of a particular pond being selected is determined solely by the likelihood of the pond being noticed, which is likely to be related to its area. This suggests the choice

$$
p_{i}=\frac{\left|\Omega_{i}\right|}{|\Omega|}=\frac{\left|\Omega_{i}\right|}{\sum_{i=1}^{n}\left|\Omega_{i}\right|}
$$

which asserts that the probability of pond $i$ being selected is its area divided by the total area of all ponds, so that larger ponds are more likely to be noticed. In this paper, we will also look at pond selection based on the likelihood of larvae surviving to adulthood on each of the various ponds. There is evidence (Spencer et al. [18]) to suggest that this is what happens in nature. One could easily imagine other more complicated choices for the $p_{i}$, perhaps taking account of density effects or geometrical factors such as apparent area rather than true area, since a pond some distance away will look smaller.

Using (2.6) and (2.4), (2.5) becomes

$$
\begin{aligned}
\frac{\partial J_{i}(t, \mathbf{x})}{\partial t}= & d_{i} \nabla^{2} J_{i}(t, \mathbf{x})-\mu_{i} J_{i}(t, \mathbf{x}) \\
& +\frac{1}{\left|\Omega_{i}\right|} b_{i}\left(p_{i} A(t)\right)-\frac{e^{-\mu_{i} \tau_{i}}}{\left|\Omega_{i}\right|} b_{i}\left(p_{i} A\left(t-\tau_{i}\right)\right) \int_{\Omega_{i}} G_{i}\left(\tau_{i}, \mathbf{x}, \mathbf{y}\right) d \mathbf{y} .
\end{aligned}
$$

Unusually for model derivations of this kind, in (2.8) we end up integrating only the known Green's function. This is because (2.6) is independent of $\mathbf{x}$, an unusual situation. In the case of the homogeneous Neumann problem the integral in (2.8) is in fact 1, as noted earlier. This is not so for the Dirichlet problem. From (2.8) we can identify the maturation rate per unit area for pond $i$, which is just the last term. This has to be integrated with respect to $\mathbf{x}$ over $\Omega_{i}$ to get an overall maturation rate for pond $i$ which is

$$
e^{-\mu_{i} \tau_{i}} b_{i}\left(p_{i} A\left(t-\tau_{i}\right)\right)
$$

for the homogeneous Neumann problem, and more generally

$$
\frac{e^{-\mu_{i} \tau_{i}}}{\left|\Omega_{i}\right|} b_{i}\left(p_{i} A\left(t-\tau_{i}\right)\right) \int_{\Omega_{i}} \int_{\Omega_{i}} G_{i}\left(\tau_{i}, \mathbf{x}, \mathbf{y}\right) d \mathbf{y} d \mathbf{x}
$$


which encompasses both homogeneous Neumann and homogeneous Dirichlet boundary conditions. We can now write down an evolution equation for the total number of adults $A(t)$. The overall maturation rate is obtained by summing the maturation rates for the individual ponds. The death rate of adults is taken as $d(A(t))$ for a suitable function $d(\cdot)$. For the homogeneous Neumann problem,

$$
\frac{d A(t)}{d t}=\sum_{i=1}^{n} e^{-\mu_{i} \tau_{i}} b_{i}\left(p_{i} A\left(t-\tau_{i}\right)\right)-d(A(t))
$$

More generally, and for the homogeneous Dirichlet case in particular,

$$
\frac{d A(t)}{d t}=\sum_{i=1}^{n} \frac{e^{-\mu_{i} \tau_{i}}}{\left|\Omega_{i}\right|} b_{i}\left(p_{i} A\left(t-\tau_{i}\right)\right) \int_{\Omega_{i}} \int_{\Omega_{i}} G_{i}\left(\tau_{i}, \mathbf{x}, \mathbf{y}\right) d \mathbf{y} d \mathbf{x}-d(A(t)) .
$$

Note that both of these equations are autonomous and feature only the adult population $A(t)$. They each contain many delays, one associated with each pond, and the delays feature in the exponential coefficients as well as the arguments of $A$, and also of the Green's functions $G_{i}(a, \mathbf{x}, \mathbf{y})$. The Green's functions contain information about the diffusivities $d_{i}$ of the juveniles in the various ponds, and of course they are also influenced by the geometry of the ponds themselves (or their patch representatives) and by the boundary conditions.

In the rest of this paper we shall focus on (2.12) which encompasses both the homogeneous Dirichlet and Neumann problems. The latter arises as a particular case of (2.12) by formally setting the double integral equal to $\left|\Omega_{i}\right|$. We begin by establishing results for (2.12) as it stands, since substantial progress is possible even when the ponds or their patch representatives are general domains $\Omega_{i}$. Later we specialise to the case when they are squares of various sizes or have habitable zones that we believe may be represented by square patches. This enables the Green's functions to be explicitly computed which is helpful for the ecological interpretation of the results.

\section{Results for the general model}

\subsection{Positivity and boundedness}

We establish the minimal assumptions needed to ensure positivity and boundedness of solutions of (2.12). The results of this section are valid for ponds or patch representatives $\Omega_{i}$ of general shapes and sizes.

Proposition 3.1 Suppose that $b_{i}(0)=0$ and $b_{i}(A) \geq 0$ for each $i$ and all $A>0$, and that $d(0)=0$. Then the solution of (2.12) satisfies $A(t) \geq 0$ for all $t>0$ if $A(\theta) \geq 0$ for all $\theta \in[-\tau, 0]$, with $\tau=\max \left(\tau_{1}, \ldots, \tau_{n}\right)$.

Suppose, in addition, that $b_{i}(A)>0$ for each $i$ and all $A>0$. Then, if $A(\theta) \geq 0$ and $A(\theta) \not \equiv 0$ on $[-\tau, 0]$, it follows that $A(t)$ becomes strictly positive at some time, and remains so thereafter. 
Proof. The first statement follows immediately from Theorem 5.2.1 of Smith [17]. To prove the second statement, suppose for a contradiction that $A(t) \equiv 0$ on $[0, \tau]$. Then from (2.12) and using the fact that the double integrals are strictly positive,

$$
b_{i}\left(p_{i} A\left(t-\tau_{i}\right)\right) \equiv 0
$$

for all $t \in[0, \tau]$, for each $i$. This is true in particular for the $i$ such that $\tau_{i}=\tau$. With that particular $i$, positive-definiteness of $b_{i}(\cdot)$ gives us $A(t-\tau) \equiv 0$ for $t \in[0, \tau]$, and this contradicts the assumption $A(\theta) \not \equiv 0$ on $[-\tau, 0]$. So $A(t)$ becomes strictly positive at some time in $[0, \tau]$, and must remain strictly positive thereafter since it satisfies $A^{\prime}(t) \geq-d(A(t))$.

Proposition 3.2 Suppose that $b_{i}(0)=0$ and $b_{i}(A) \geq 0$ for each $i$ and all $A>0$, and $\sup _{A \in[0, \infty)} b_{i}(A)<\infty$ for each $i$. Suppose that $d(0)=0$ and that $d(A)$ is a strictly increasing function which grows large enough so that the right hand side of the following inequality (3.13) is finite. Then the solution of (2.12) satisfies

$$
\limsup _{t \rightarrow \infty} A(t) \leq d^{-1}\left(\sum_{i=1}^{n} \frac{e^{-\mu_{i} \tau_{i}}}{\left|\Omega_{i}\right|} \sup _{A \geq 0} b_{i}(A) \int_{\Omega_{i}} \int_{\Omega_{i}} G_{i}\left(\tau_{i}, \mathbf{x}, \mathbf{y}\right) d \mathbf{y} d \mathbf{x}\right) .
$$

Proof. From (2.12),

$$
\frac{d A(t)}{d t} \leq \sum_{i=1}^{n} \frac{e^{-\mu_{i} \tau_{i}}}{\left|\Omega_{i}\right|} \sup _{A \geq 0} b_{i}(A) \int_{\Omega_{i}} \int_{\Omega_{i}} G_{i}\left(\tau_{i}, \mathbf{x}, \mathbf{y}\right) d \mathbf{y} d \mathbf{x}-d(A(t))
$$

so, from a simple comparison argument, $A(t)$ is bounded by the solution of the differential equation we get from (3.14) on replacing $\leq$ by $=$. Then, (3.13) follows immediately using the properties of $d(\cdot)$.

\subsection{Linear stability of the extinction state}

In this section and the next we consider in more detail the evolution of the total adult population $A(t)$ in the case when homogeneous Dirichlet boundary conditions are applied on the boundaries of patches $\Omega_{i}$ that represent the habitable zones of the actual ponds. In particular we focus on conditions which distinguish between the outcomes of extinction or persistence. Linearising (2.12) about the extinction state, when $A(t) \equiv 0$, we obtain

$$
\frac{d A(t)}{d t}=\sum_{i=1}^{n} \frac{p_{i} e^{-\mu_{i} \tau_{i}}}{\left|\Omega_{i}\right|} b_{i}^{\prime}(0) A\left(t-\tau_{i}\right) \int_{\Omega_{i}} \int_{\Omega_{i}} G_{i}\left(\tau_{i}, \mathbf{x}, \mathbf{y}\right) d \mathbf{y} d \mathbf{x}-d^{\prime}(0) A(t) .
$$

Seeking solutions of the form $A(t)=\exp (\lambda t)$ yields the characteristic equation

$$
\lambda+d^{\prime}(0)=\sum_{i=1}^{n} \frac{p_{i} e^{-\mu_{i} \tau_{i}}}{\left|\Omega_{i}\right|} b_{i}^{\prime}(0) e^{-\lambda \tau_{i}} \int_{\Omega_{i}} \int_{\Omega_{i}} G_{i}\left(\tau_{i}, \mathbf{x}, \mathbf{y}\right) d \mathbf{y} d \mathbf{x} .
$$


If $b_{i}^{\prime}(0)>0$ for each $i$ then the coefficients of the delay terms in (3.15) are all positive, and this allows us to invoke Theorem 5.5.1 of Smith [17] to conclude that the dominant root of the characteristic equation (3.16) is real, so that only real roots of the equation need be considered. Moreover, the right hand side of (3.16) is a decreasing function of the real variable $\lambda$ while the left hand side is strictly increasing. Therefore (3.16) can only have one real root, which is negative if the left hand side of (3.16) exceeds the right hand side when $\lambda=0$, i.e. if

$$
d^{\prime}(0)>\sum_{i=1}^{n} \frac{p_{i} e^{-\mu_{i} \tau_{i}}}{\left|\Omega_{i}\right|} b_{i}^{\prime}(0) \int_{\Omega_{i}} \int_{\Omega_{i}} G_{i}\left(\tau_{i}, \mathbf{x}, \mathbf{y}\right) d \mathbf{y} d \mathbf{x}
$$

and is positive if the reverse inequality holds. We have the following theorem. The fact that each $J_{i}(t, \mathbf{x})$ tends to zero under condition (3.17) follows from the asymptotically autonomous limiting form of (2.8) in the limit when $t \rightarrow \infty$ and $A(t) \rightarrow 0$, and the application of Theorem 4.2 in Thieme [19].

Theorem 3.3 Suppose the birth and death functions are differentiable and satisfy $b_{i}(0)=0, b_{i}^{\prime}(0)>0$ for each $i, d(0)=0$ and $d^{\prime}(0)>0$. If (3.17) holds then $A(t) \rightarrow 0$ and each $J_{i}(t, \mathbf{x}) \rightarrow 0$ as $t \rightarrow \infty$ as solutions of the linearised problem.

Later, we will discuss in detail how to interpret Theorem 3.3 for particular choices of the probabilities $p_{i}$ and for the case when the $\Omega_{i}$ are squares of various sizes.

\subsection{Persistence}

In this section we prove persistence for the variable $A(t)$ in the uniform sense, where $A(t)$ satisfies (2.12). Uniform persistence holds when there exists a constant $\eta>0$, which is independent of the initial conditions, such that,

$$
\liminf _{t \rightarrow \infty} A(t) \geq \eta
$$

We establish this persistence property using Theorem 4.1 of Hale and Waltman [12]. Let a metric space $X$ be the closure of an open set $X^{0}$, so that $X=X^{0} \cup \partial X^{0}$ where $\partial X^{0}$ is the boundary of $X^{0}$. Let $T(t)$ be a $C^{0}$-semigroup on $X$ satisfying

$$
T(t): X^{0} \rightarrow X^{0}, \quad T(t): \partial X^{0} \rightarrow \partial X^{0} .
$$

Suppose that the restricted semiflow has the global attractor $A_{\partial}$ in $\partial X^{0}$, and assume that

$$
\tilde{A}_{\partial}=\bigcup_{x \in A_{\partial}} \omega(x)
$$

where $\omega(x)$ is the $\omega$-limit set of $x$. Then the following holds (Hale and Walt$\operatorname{man}[12])$ :

Theorem 3.4 (Hale and Waltman) Suppose that $T(t)$ satisfies (3.19) and that 
(i) there is a $t_{0} \geq 0$ such that $T(t)$ is compact for $t>t_{0}$;

(ii) $T(t)$ is point dissipative in $X$;

(iii) $\tilde{A}_{\partial}$ is isolated and has an acyclic covering $M$.

Then $T(t)$ is uniformly persistent if and only if for each $M_{i} \in M$,

$$
W^{s}\left(M_{i}\right) \cap X^{0}=\Phi .
$$

Here, $W^{s}\left(M_{i}\right)$ is the stable manifold of $M_{i}$. We take $X$ to be the non-negative cone of $C([-\tau, 0], \mathbf{R})$, where $\tau=\max \left(\tau_{1}, \ldots, \tau_{n}\right)$. Define

$$
\begin{aligned}
X^{0} & =\{\varphi \in X: \varphi(\theta)>0 \text { for some } \theta \in[-\tau, 0]\} \\
\partial X^{0} & =\{\varphi \in X: \varphi(\theta)=0 \text { for all } \theta \in[-\tau, 0]\}
\end{aligned}
$$

Then $X=X^{0} \cup \partial X^{0}$.

Theorem 3.5 Suppose the birth and death functions are continuously differentiable and satisfy all the assumptions of Propositions 3.1 and 3.2, together with $b_{i}^{\prime}(0)>0$ for each $i$, and $d^{\prime}(0)>0$. If

$$
d^{\prime}(0)<\sum_{i=1}^{n} \frac{p_{i} e^{-\mu_{i} \tau_{i}}}{\left|\Omega_{i}\right|} b_{i}^{\prime}(0) \int_{\Omega_{i}} \int_{\Omega_{i}} G_{i}\left(\tau_{i}, \mathbf{x}, \mathbf{y}\right) d \mathbf{y} d \mathbf{x}
$$

then there exists $\eta>0$ such that (3.18) holds for any non-negative initial datum that lies in $X^{0}$.

Proof. We choose $T(t): X \rightarrow X$ to be the operator which maps $A_{0}$ to $A_{t}$, where $A(t)$ satisfies (2.12) and $A_{t}$ is the function $A_{t}(\theta)=A(t+\theta), \theta \in[-\tau, 0]$. With this notation, $A_{0}$ is the function with values $A(\theta), \theta \in[-\tau, 0]$, and is the initial datum for (2.12). So $T(t)$ maps the initial datum to the state of the variable $A$ at time $t$, which comprises its values on the interval $[t-\tau, t]$.

Now, the sole member of $\partial X^{0}$ is the function which, when used as the initial datum for (2.12), results in the solution of that equation remaining zero for all $t>0$, so clearly $T(t): \partial X^{0} \rightarrow \partial X^{0}$. Let us now show that $T(t): X^{0} \rightarrow X^{0}$. Select a function in $X^{0}$. Then, by Proposition 3.1, $A(t)$ becomes strictly positive at some future time and remains so. If the state $A_{t}$ were ever to leave $X^{0}$ and arrive at $\partial X^{0}$ at some time $t^{*}>0$, the implication is that $A(t) \equiv 0$ for $t \in\left[t^{*}-\tau, t^{*}\right]$. But the only solution of (2.12) for $t>t^{*}$ which satisfies this is $A(t) \equiv 0, t>t^{*}$, and this contradicts the fact that the solution of (2.12) must become and remain strictly positive for initial data in $X^{0}$. So $T(t): X^{0} \rightarrow X^{0}$ and (3.19) holds.

Condition (i) of Theorem 3.4 holds for any $t_{0}>\tau$. Condition (ii) holds because of the boundedness (Proposition 3.2). Condition (iii) holds trivially (the set $M$ here is just the zero equilibrium of (2.12)). All that needs to be checked is that $W^{s}(0) \cap X^{0}=\Phi$, where $W^{s}(0)$ is the stable manifold of the equilibrium $A \equiv 0$ of (2.12). We prove this by contradiction. Suppose a solution of (2.12) exists 
starting in $X^{0}$ and in $W^{s}(0)$. Since the initial datum for this solution is in $X^{0}$, by Proposition 3.1 the solution must become and remain strictly positive. However since the initial datum is also in $W^{s}(0)$ the solution $A(t)$ must approach 0 . This convergence to 0 may or may not be monotone, but in all cases there exists a sequence of times $t_{j} \rightarrow \infty$ such that $A\left(t_{j}\right) \rightarrow 0$ as $j \rightarrow \infty, A^{\prime}\left(t_{j}\right) \leq 0$ and $A\left(t_{j}\right) \leq A(t)$ for all $t \leq t_{j}$. Evaluating (2.12) at $t=t_{j}$ gives

$$
\begin{aligned}
\frac{d A\left(t_{j}\right)}{d t} & =\sum_{i=1}^{n} \frac{e^{-\mu_{i} \tau_{i}}}{\left|\Omega_{i}\right|} b_{i}\left(p_{i} A\left(t_{j}-\tau_{i}\right)\right) \int_{\Omega_{i}} \int_{\Omega_{i}} G_{i}\left(\tau_{i}, \mathbf{x}, \mathbf{y}\right) d \mathbf{y} d \mathbf{x}-d\left(A\left(t_{j}\right)\right) \\
& =\sum_{i=1}^{n} \frac{p_{i} e^{-\mu_{i} \tau_{i}}}{\left|\Omega_{i}\right|} b_{i}^{\prime}\left(\Theta_{i}\left(t_{j}\right)\right) A\left(t_{j}-\tau_{i}\right) \int_{\Omega_{i}} \int_{\Omega_{i}} G_{i}\left(\tau_{i}, \mathbf{x}, \mathbf{y}\right) d \mathbf{y} d \mathbf{x}-d^{\prime}\left(\Psi\left(t_{j}\right)\right) A\left(t_{j}\right)
\end{aligned}
$$

using the mean value theorem, where $\Theta_{i}(t) \in\left(0, p_{i} A\left(t-\tau_{i}\right)\right)$ and $\Psi(t) \in(0, A(t))$. Since $A(t) \rightarrow 0, \Theta_{i}\left(t_{j}\right) \rightarrow 0$ as $j \rightarrow \infty$ and therefore $b_{i}^{\prime}\left(\Theta_{i}\left(t_{j}\right)\right)>0$ for $j$ sufficiently large. For such $j$,

$$
\frac{d A\left(t_{j}\right)}{d t} \geq \sum_{i=1}^{n} \frac{p_{i} e^{-\mu_{i} \tau_{i}}}{\left|\Omega_{i}\right|} b_{i}^{\prime}\left(\Theta_{i}\left(t_{j}\right)\right) A\left(t_{j}\right) \int_{\Omega_{i}} \int_{\Omega_{i}} G_{i}\left(\tau_{i}, \mathbf{x}, \mathbf{y}\right) d \mathbf{y} d \mathbf{x}-d^{\prime}\left(\Psi\left(t_{j}\right)\right) A\left(t_{j}\right) .
$$

Since the solution becomes and remains strictly positive, $A\left(t_{j}\right)>0$ for sufficiently large $j$. Therefore

$$
\frac{A^{\prime}\left(t_{j}\right)}{A\left(t_{j}\right)} \geq \sum_{i=1}^{n} \frac{p_{i} e^{-\mu_{i} \tau_{i}}}{\left|\Omega_{i}\right|} b_{i}^{\prime}\left(\Theta_{i}\left(t_{j}\right)\right) \int_{\Omega_{i}} \int_{\Omega_{i}} G_{i}\left(\tau_{i}, \mathbf{x}, \mathbf{y}\right) d \mathbf{y} d \mathbf{x}-d^{\prime}\left(\Psi\left(t_{j}\right)\right) .
$$

Taking the limit as $j \rightarrow \infty$ and using $\Theta_{i}\left(t_{j}\right), \Psi\left(t_{j}\right) \rightarrow 0$ gives

$$
\begin{aligned}
\liminf _{j \rightarrow \infty} \frac{A^{\prime}\left(t_{j}\right)}{A\left(t_{j}\right)} & \geq \sum_{i=1}^{n} \frac{p_{i} e^{-\mu_{i} \tau_{i}}}{\left|\Omega_{i}\right|} b_{i}^{\prime}(0) \int_{\Omega_{i}} \int_{\Omega_{i}} G_{i}\left(\tau_{i}, \mathbf{x}, \mathbf{y}\right) d \mathbf{y} d \mathbf{x}-d^{\prime}(0) \\
& >0
\end{aligned}
$$

by (3.22). This implies $A^{\prime}\left(t_{j}\right)>0$ for $j$ sufficiently large, which contradicts $A^{\prime}\left(t_{j}\right) \leq 0$.

\section{Pond selection based only on area}

If pond selection is based solely on area then the probabilities $p_{i}$ are chosen as in (2.7) and (2.12) becomes

$$
\frac{d A(t)}{d t}=\sum_{i=1}^{n} \frac{e^{-\mu_{i} \tau_{i}}}{\left|\Omega_{i}\right|} b_{i}\left(\frac{\left|\Omega_{i}\right|}{|\Omega|} A\left(t-\tau_{i}\right)\right) \int_{\Omega_{i}} \int_{\Omega_{i}} G_{i}\left(\tau_{i}, \mathbf{x}, \mathbf{y}\right) d \mathbf{y} d \mathbf{x}-d(A(t)) .
$$


We consider in more detail the evolution of the total adult population $A(t)$ satisfying (4.23) in the case when homogeneous Dirichlet boundary conditions are applied on the boundaries of the patches $\Omega_{i}$ representing the ponds. In order to interpret the conditions ecologically it is very useful to be able to evaluate explicitly the integral of $G_{i}\left(\tau_{i}, \mathbf{x}, \mathbf{y}\right)$ in $(4.23)$, which is possible only for particular, simple patches. We shall focus on the case when the patches are squares of various sizes, so that $\Omega_{i}=\left(0, l_{i}\right) \times\left(0, l_{i}\right), i=1,2, \ldots, n$. For square patches, $\left|\Omega_{i}\right|=l_{i}^{2}$ and $|\Omega|=\sum_{i=1}^{n} l_{i}^{2}$. In the case of homogeneous Dirichlet boundary conditions, and letting $\mathbf{x}=\left(x_{1}, x_{2}\right), \mathbf{y}=\left(y_{1}, y_{2}\right)$, we find that the Green's functions $G_{i}(a, \mathbf{x}, \mathbf{y})$ turn out to be given by

$$
G_{i}(a, \mathbf{x}, \mathbf{y})=\frac{4}{l_{i}^{2}} \sum_{k=1}^{\infty} \sum_{m=1}^{\infty} e^{-d_{i} \pi^{2}\left(k^{2}+m^{2}\right) a / l_{i}^{2}} \sin \frac{k \pi x_{1}}{l_{i}} \sin \frac{m \pi x_{2}}{l_{i}} \sin \frac{k \pi y_{1}}{l_{i}} \sin \frac{m \pi y_{2}}{l_{i}}
$$

After some algebra,

$$
\int_{\Omega_{i}} \int_{\Omega_{i}} G_{i}\left(\tau_{i}, \mathbf{x}, \mathbf{y}\right) d \mathbf{y} d \mathbf{x}=\left(\frac{8 l_{i}}{\pi^{2}} \sum_{k=0}^{\infty} \frac{1}{(2 k+1)^{2}} e^{-d_{i} \pi^{2}(2 k+1)^{2} \tau_{i} / l_{i}^{2}}\right)^{2} .
$$

We have the following theorem on the stability of the extinction steady state to small perturbations. It is a particular case of Theorem 3.3.

Theorem 4.1 Suppose the birth and death functions are differentiable and satisfy $b_{i}(0)=0, b_{i}^{\prime}(0)>0$ for each $i, d(0)=0$ and $d^{\prime}(0)>0$. Suppose that $\Omega_{i}=$ $\left(0, l_{i}\right) \times\left(0, l_{i}\right), i=1,2, \ldots, n$ and that the $J_{i}(t, \mathbf{x})$ satisfy homogeneous Dirichlet boundary conditions on $\partial \Omega_{i}$ for each $i$. If

$$
d^{\prime}(0) \sum_{i=1}^{n} l_{i}^{2}>\sum_{i=1}^{n} e^{-\mu_{i} \tau_{i}} b_{i}^{\prime}(0) \frac{64 l_{i}^{2}}{\pi^{4}}\left(\sum_{k=0}^{\infty} \frac{1}{(2 k+1)^{2}} e^{-d_{i} \pi^{2}(2 k+1)^{2} \tau_{i} / l_{i}^{2}}\right)^{2}
$$

then the solution of the linearisation of (4.23) satisfies $A(t) \rightarrow 0$. Moreover each $J_{i}(t, \mathbf{x}) \rightarrow 0$ as $t \rightarrow \infty$.

From Theorem 4.1 we can make a number of observations regarding minimum and/or maximum allowable sizes for the square patches (and therefore for predatorinfested ponds with hostile interiors) if the mosquitoes are to survive. First let us consider what happens if we scale all patches by the same linear factor $\lambda$, so that each $l_{i}$ is replaced by $\lambda l_{i}$. In inequality (4.26) such a replacement results in $\lambda$ appearing only in the argument of the exponential, since all other $\lambda$ 's will cancel. It is easily seen that there exists some critical value of $\lambda$, below which the outcome is extinction. So if the patches/ponds are uniformly scaled down in size, extinction will result. An analogous result is well known to hold for simple scalar reactiondiffusion equations on bounded domains with homogeneous Dirichlet boundary conditions, such as the well known KISS model (see Okubo and Levin [16]). 
Of greater interest is the question of whether increasing some of the patch sizes, leaving others unchanged, could also drive the population to extinction. The answer to this question is yes, and can be seen from inequality (4.26). Imagine we increase the size of $\Omega_{1}$, leaving the other patches unchanged. This corresponds to increasing $l_{1}$. Since

$$
\sum_{k=0}^{\infty} \frac{1}{(2 k+1)^{2}} e^{-d_{i} \pi^{2}(2 k+1)^{2} \tau_{i} / l_{i}^{2}}<\sum_{k=0}^{\infty} \frac{1}{(2 k+1)^{2}}=\frac{\pi^{2}}{8}
$$

it is easily seen that if $l_{1}$ is sufficiently large then inequality (4.26) will hold, implying extinction, if $d^{\prime}(0)>b_{1}^{\prime}(0) e^{-\mu_{1} \tau_{1}}$. This inequality states that, at low densities, the per-capita death rate exceeds the per-capita maturation rate for patch 1 (the latter being the per-capita birth rate for patch 1 multiplied by the probability of survival to maturation on that patch). So, if conditions at patch 1 are not conducive to survival, and if patch 1 is sufficiently large relative to other patches, then extinction is the outcome for the whole population on $n$ patches. Recall that we are assuming the adults are more likely to choose large ponds for egg laying. In the scenario being described here, patch 1 is large and the corresponding pond will attract a large number of adults for egg laying, but it turns out to be a poor choice of pond because it offers low survival prospects for larvae.

Theorem 3.5 on persistence becomes

Theorem 4.2 Suppose the birth and death functions are continuously differentiable and satisfy all the assumptions of Propositions 3.1 and 3.2, together with $b_{i}^{\prime}(0)>0$ for each $i$, and $d^{\prime}(0)>0$. Suppose that $\Omega_{i}=\left(0, l_{i}\right) \times\left(0, l_{i}\right), i=1,2, \ldots, n$ with the $J_{i}(t, \mathbf{x})$ satisfying homogeneous Dirichlet boundary conditions on $\partial \Omega_{i}$ for each $i$. If $A(t)$ satisfies (4.23) and

$$
d^{\prime}(0) \sum_{i=1}^{n} l_{i}^{2}<\sum_{i=1}^{n} e^{-\mu_{i} \tau_{i}} b_{i}^{\prime}(0) \frac{64 l_{i}^{2}}{\pi^{4}}\left(\sum_{k=0}^{\infty} \frac{1}{(2 k+1)^{2}} e^{-d_{i} \pi^{2}(2 k+1)^{2} \tau_{i} / l_{i}^{2}}\right)^{2}
$$

holds, then there exists $\eta>0$ such that $\liminf _{t \rightarrow \infty} A(t) \geq \eta$ for any non-negative initial datum that lies in $X^{0}$.

\section{Pond selection based on both larval survival probability and area}

In the previous section we considered the situation where a female adult mosquito bases her choice of oviposition pond only on the size of the pond relative to the total size of all ponds, so that her choice of a particular pond is determined purely by the likelihood of the pond being noticed, which we assumed would be determined by the size of the pond as measured by area. Such an assumption 
ignores the possibility that a large pond might not in fact be a good one for oviposition. Indeed, as we have just noted, a very large pond in which survival prospects for larvae are poor could result in extinction for the entire population. As noted earlier, mosquitoes try to avoid ovipositing in ponds containing predators of larvae (Spencer et al. [18]). The larvae of Culiseta longiareolata are vulnerable to predation by the backswimmer Nononecta maculata. To a first approximation, it seems reasonable to assume that the number of $N$. maculata in a particular pond is constant, since most ponds have abundant alternative prey that can support $N$. maculata in the absence of the larvae of $C$. longiareolata [18]. The number could differ between ponds, however. With this assumption, the effect of predation of larval mosquitoes in pond $i$ can be accounted for simply by modifying the value of $\mu_{i}$. Knowledge of the presence and number of predators in a particular pond corresponds to knowledge of the likelihood of a larva surviving to maturation in that pond, and can therefore be modelled by taking the probability $p_{i}$, of selecting pond $i$ for oviposition, to depend on the probability of successful maturation in that pond which is $P_{i}$, defined by

$$
P_{i}=\frac{e^{-\mu_{i} \tau_{i}}}{\left|\Omega_{i}\right|} \int_{\Omega_{i}} \int_{\Omega_{i}} G_{i}\left(\tau_{i}, \mathbf{x}, \mathbf{y}\right) d \mathbf{y} d \mathbf{x} .
$$

All this is very much a first approximation. The reality need not be so simple. Although it is known that $C$. longiareolata can detect the presence of $N$. maculata in a pond at very low densities, it is not clear whether the adult mosquitoes are able to assess the level of predation risk (Eitam and Blaustein [9]) and adjust their behaviour accordingly. Though the larval survival probability for a particular pond would depend on the number of predators in that pond, adult mosquitoes choosing a pond for oviposition would not necessarily use this information in the simple way we assume here. If they cannot assess the degree of risk, they may take the simpler view that either a pond contains predators or it does not. Also, mosquitoes may not have time to visit all ponds before choosing, and may select a pond with a high number of predators even though more suitable ponds are available.

Part of expression (5.28), $e^{-\mu_{i} \tau_{i}}$, is the probability that a larva survives to maturation in pond $i$ in the absence of any chance of death due to boundary effects, i.e. reaching the hostile interior of the pond. The probability of not dying such a death is the remaining part of the expression, namely $\int_{\Omega_{i}} \int_{\Omega_{i}} G_{i}\left(\tau_{i}, \mathbf{x}, \mathbf{y}\right) d \mathbf{y} d \mathbf{x} /\left|\Omega_{i}\right|$. The latter expression equals 1 in the case of homogeneous Neumann boundary conditions. For homogeneous Dirichlet boundary conditions it is some number less than 1 which monotonically increases and approaches 1 as the patch $\Omega_{i}$ representing a pond grows in size. To see this, suppose for the moment that $\Omega_{i}$ is some patch and introduce a scaling parameter $\lambda>0$ such that $\Omega_{i}^{\lambda}$ has the same shape as $\Omega_{i}$ but is scaled in size by a factor of $\lambda$. For example, if $\Omega_{i}=(0,1) \times(0,1)$ then $\Omega_{i}^{\lambda}=(0, \lambda) \times(0, \lambda)$. Let $\mathbf{x}^{\lambda}=\lambda \mathbf{x}, \mathbf{y}^{\lambda}=\lambda \mathbf{y}$, and let $G_{i}^{\lambda}\left(a, \mathbf{x}^{\lambda}, \mathbf{y}^{\lambda}\right)$ denote the Green's function for $\partial G_{i}^{\lambda} / \partial a=d_{i} \nabla_{\mathbf{x}^{\lambda}}^{2} G_{i}^{\lambda}$ in $\Omega_{i}^{\lambda}$ which, for Dirichlet boundary conditions, satisfies $G_{i}^{\lambda}=0$ on $\partial \Omega_{i}^{\lambda}$. We have the following result. 
Proposition 5.1 (i) Under homogeneous Neumann boundary conditions,

$$
\frac{1}{\left|\Omega_{i}\right|} \int_{\Omega_{i}} \int_{\Omega_{i}} G_{i}\left(\tau_{i}, \mathbf{x}, \mathbf{y}\right) d \mathbf{y} d \mathbf{x}=1
$$

(ii) Under homogeneous Dirichlet boundary conditions,

$$
\frac{1}{\left|\Omega_{i}^{\lambda}\right|} \int_{\Omega_{i}^{\lambda}} \int_{\Omega_{i}^{\lambda}} G_{i}^{\lambda}\left(\tau_{i}, \mathbf{x}^{\lambda}, \mathbf{y}^{\lambda}\right) d \mathbf{y}^{\lambda} d \mathbf{x}^{\lambda}
$$

is an increasing function of $\lambda$ which approaches 1 as $\lambda \rightarrow \infty$.

Proof. The conclusion in (i) can be shown directly. We now show (ii). Now, $G_{i}$, the Green's function associated with $\Omega_{i}$, satisfies $(2.3)$ and $G_{i}=0$ on $\partial \Omega_{i}$. Also

$$
\left|\Omega_{i}^{\lambda}\right|=\int_{\Omega_{i}^{\lambda}} d x_{1}^{\lambda} d x_{2}^{\lambda}=\lambda^{2} \int_{\Omega_{i}} d x_{1} d x_{2}=\lambda^{2}\left|\Omega_{i}\right|
$$

and

$$
\int_{\Omega_{i}^{\lambda}} \int_{\Omega_{i}^{\lambda}} G_{i}^{\lambda}\left(\tau_{i}, \mathbf{x}^{\lambda}, \mathbf{y}^{\lambda}\right) d \mathbf{y}^{\lambda} d \mathbf{x}^{\lambda}=\lambda^{4} \int_{\Omega_{i}} \int_{\Omega_{i}} G_{i}^{\lambda}\left(\tau_{i}, \lambda \mathbf{x}, \lambda \mathbf{y}\right) d \mathbf{y} d \mathbf{x}
$$

Scaling arguments show that

$$
G_{i}^{\lambda}(a, \mathbf{x}, \mathbf{y})=\frac{1}{\lambda^{2}} G_{i}\left(a / \lambda^{2}, \mathbf{x} / \lambda, \mathbf{y} / \lambda\right)
$$

where $G_{i}$ satisfies (2.3). Hence

$$
\begin{aligned}
\frac{1}{\left|\Omega_{i}^{\lambda}\right|} \int_{\Omega_{i}^{\lambda}} \int_{\Omega_{i}^{\lambda}} G_{i}^{\lambda}\left(\tau_{i}, \mathbf{x}^{\lambda}, \mathbf{y}^{\lambda}\right) d \mathbf{y}^{\lambda} d \mathbf{x}^{\lambda} & =\frac{\lambda^{2}}{\left|\Omega_{i}\right|} \int_{\Omega_{i}} \int_{\Omega_{i}} G_{i}^{\lambda}\left(\tau_{i}, \lambda \mathbf{x}, \lambda \mathbf{y}\right) d \mathbf{y} d \mathbf{x} \\
& =\frac{1}{\left|\Omega_{i}\right|} \int_{\Omega_{i}} \int_{\Omega_{i}} G_{i}\left(\tau_{i} / \lambda^{2}, \mathbf{x}, \mathbf{y}\right) d \mathbf{y} d \mathbf{x} \\
& =: \frac{1}{\left|\Omega_{i}\right|} f\left(\lambda^{2}\right) .
\end{aligned}
$$

Our claim follows if $f(\infty)=\left|\Omega_{i}\right|$ and $f$ is increasing. But

$$
f(\infty)=\int_{\Omega_{i}} \int_{\Omega_{i}} G_{i}(0, \mathbf{x}, \mathbf{y}) d \mathbf{y} d \mathbf{x}=\int_{\Omega_{i}} \int_{\Omega_{i}} \delta(\mathbf{x}-\mathbf{y}) d \mathbf{y} d \mathbf{x}=\int_{\Omega_{i}} d \mathbf{x}=\left|\Omega_{i}\right|
$$

and

$$
\begin{aligned}
\frac{d f\left(\lambda^{2}\right)}{d\left(\lambda^{2}\right)} & =-\frac{1}{\lambda^{4}} \int_{\Omega_{i}} \int_{\Omega_{i}} d_{i} \nabla_{\mathbf{x}}^{2} G_{i}\left(\tau_{i} / \lambda^{2}, \mathbf{x}, \mathbf{y}\right) d \mathbf{y} d \mathbf{x} \\
& =-\frac{1}{\lambda^{4}} \int_{\Omega_{i}}\left(\int_{\partial \Omega_{i}} d_{i} \nabla_{\mathbf{x}} G_{i}\left(\tau_{i} / \lambda^{2}, \mathbf{x}, \mathbf{y}\right) \cdot \mathbf{n} d s\right) d \mathbf{x}
\end{aligned}
$$


using Green's theorem to convert the inner integral to a line integral taken around the boundary $\partial \Omega_{i}$ of $\Omega_{i}$, where $d s$ is the increment of arc-length along $\partial \Omega_{i}$ and $\mathbf{n}$ is the outward pointing unit normal. But $\nabla_{\mathbf{x}} G_{i}\left(\tau_{i} / \lambda^{2}, \mathbf{x}, \mathbf{y}\right) \cdot \mathbf{n} \leq 0$ on $\partial \Omega_{i}$, since the Green's function must satisfy $G_{i} \geq 0$ in $\Omega_{i}$ and $G_{i}=0$ on $\partial \Omega_{i}$ in the Dirichlet case. Hence $f$ is indeed increasing.

We propose to take account of the female adult mosquito's tendency to avoid ovipositing in ponds containing predators by supposing that the probability of her choice falling on pond $i$ is proportional to the probability of a larva born in that pond surviving to maturation. This probability is (5.28) and, as we have just noted, it increases with the area of the pond unless the boundary conditions on the patch representing it are homogeneous Neumann (i.e. the pond has no hostile interior) in which case the probability is $e^{-\mu_{i} \tau_{i}}$ irrespective of area. In view of the latter observation, it seems reasonable to suppose that pond size as measured by area should still play a direct role in pond selection otherwise the implication is that mosquitoes would always prefer a very tiny pond if it happened to offer larvae a slightly higher prospect of survival. For this reason, we propose that the probability $p_{i}$ of pond $i$ being selected for oviposition should be proportional both to $\left|\Omega_{i}\right|$ and to expression (5.28). These assumptions lead to

$$
p_{i}=\frac{e^{-\mu_{i} \tau_{i}} \int_{\Omega_{i}} \int_{\Omega_{i}} G_{i}\left(\tau_{i}, \mathbf{x}, \mathbf{y}\right) d \mathbf{y} d \mathbf{x}}{\sum_{i=1}^{n} e^{-\mu_{i} \tau_{i}} \int_{\Omega_{i}} \int_{\Omega_{i}} G_{i}\left(\tau_{i}, \mathbf{x}, \mathbf{y}\right) d \mathbf{y} d \mathbf{x}}
$$

and to the model

$$
\begin{aligned}
\frac{d A(t)}{d t}= & \sum_{i=1}^{n} \frac{e^{-\mu_{i} \tau_{i}}}{\left|\Omega_{i}\right|} b_{i}\left(\frac{e^{-\mu_{i} \tau_{i}} \int_{\Omega_{i}} \int_{\Omega_{i}} G_{i}\left(\tau_{i}, \mathbf{x}, \mathbf{y}\right) d \mathbf{y} d \mathbf{x}}{\sum_{i=1}^{n} e^{-\mu_{i} \tau_{i}} \int_{\Omega_{i}} \int_{\Omega_{i}} G_{i}\left(\tau_{i}, \mathbf{x}, \mathbf{y}\right) d \mathbf{y} d \mathbf{x}} A\left(t-\tau_{i}\right)\right) \\
& \times \int_{\Omega_{i}} \int_{\Omega_{i}} G_{i}\left(\tau_{i}, \mathbf{x}, \mathbf{y}\right) d \mathbf{y} d \mathbf{x}-d(A(t)) .
\end{aligned}
$$

In the case when the patches representing the ponds are the squares $\Omega_{i}=\left(0, l_{i}\right) \times$ $\left(0, l_{i}\right)$, we have the following theorem on extinction which is a particular case of Theorem 3.3.

Theorem 5.2 Suppose the birth and death functions are differentiable and satisfy $b_{i}(0)=0, b_{i}^{\prime}(0)>0$ for each $i, d(0)=0$ and $d^{\prime}(0)>0$. Suppose that $\Omega_{i}=$ $\left(0, l_{i}\right) \times\left(0, l_{i}\right), i=1,2, \ldots, n$ and that the $J_{i}(t, \mathbf{x})$ satisfy homogeneous Dirichlet boundary conditions on $\partial \Omega_{i}$ for each $i$. If

$d^{\prime}(0) \sum_{i=1}^{n} l_{i}^{2} e^{-\mu_{i} \tau_{i}}\left(\sum_{k=0}^{\infty} \frac{e^{-d_{i} \pi^{2}(2 k+1)^{2} \tau_{i} / l_{i}^{2}}}{(2 k+1)^{2}}\right)^{2}>\sum_{i=1}^{n} \frac{64 l_{i}^{2} e^{-2 \mu_{i} \tau_{i}} b_{i}^{\prime}(0)}{\pi^{4}}\left(\sum_{k=0}^{\infty} \frac{e^{-d_{i} \pi^{2}(2 k+1)^{2} \tau_{i} / l_{i}^{2}}}{(2 k+1)^{2}}\right)^{4}$

then the solution of the linearisation of (5.30) satisfies $A(t) \rightarrow 0$. Also, each $J_{i}(t, \mathbf{x}) \rightarrow 0$ as $t \rightarrow \infty$.

For persistence, one can state a theorem similar to Theorem 4.2 but relating to equation (5.30). The condition for persistence is just the reversal of the strict inequality in (5.31). 


\section{Comparison of strategies for pond selection}

The conditions in Theorems 4.1 and 5.2 relating to extinction have the advantage, in the form stated in those theorems, of enabling a discussion of the effects of spatial scale as noted earlier. For square patches it is worth noting that they can both be alternatively written in terms of the probability $P_{i}$, defined in (5.28), that a larva will survive to maturation in pond $i$. Condition (4.26) for extinction, relating to the situation when pond selection is based only on area, becomes

$$
\sum_{i=1}^{n} l_{i}^{2}\left(d^{\prime}(0)-b_{i}^{\prime}(0) P_{i}\right)>0
$$

while condition (5.31), relating to pond selection based on larval survival probability as well as area, becomes

$$
\sum_{i=1}^{n} l_{i}^{2} P_{i}\left(d^{\prime}(0)-b_{i}^{\prime}(0) P_{i}\right)>0 .
$$

It is of particular interest to ask whether there are parameter values for which the mosquito population will survive if they base their pond selection only on area, but go extinct if they additionally try to take account of expected larval survival probability in the ponds as modelled in this paper. Such an outcome would be counterintuitive, but it is a possibility. For the case of two ponds, we are asking whether it is possible to have

$$
l_{1}^{2} P_{1}\left(d^{\prime}(0)-b_{1}^{\prime}(0) P_{1}\right)+l_{2}^{2} P_{2}\left(d^{\prime}(0)-b_{2}^{\prime}(0) P_{2}\right)>0
$$

and

$$
l_{1}^{2}\left(d^{\prime}(0)-b_{1}^{\prime}(0) P_{1}\right)+l_{2}^{2}\left(d^{\prime}(0)-b_{2}^{\prime}(0) P_{2}\right)<0
$$

simultaneously. Introduce $\alpha_{1}=b_{1}^{\prime}(0) / d^{\prime}(0)$ and $\alpha_{2}=b_{2}^{\prime}(0) / d^{\prime}(0)$ and let $\epsilon>0$ be fixed. We investigate whether it is possible to have

$$
\left(\frac{l_{1}}{l_{2}}\right)^{2} P_{1}\left(1-\alpha_{1} P_{1}\right)+P_{2}\left(1-\alpha_{2} P_{2}\right)>0
$$

while

$$
\left(\frac{l_{1}}{l_{2}}\right)^{2}\left(1-\alpha_{1} P_{1}\right)+1-\alpha_{2} P_{2}=-\epsilon,
$$

i.e., while

$$
P_{2}=\frac{1}{\alpha_{2}}\left[\left(\frac{l_{1}}{l_{2}}\right)^{2}\left(1-\alpha_{1} P_{1}\right)+1+\epsilon\right]
$$

provided that the right hand side of this expression is between 0 and 1 , since $P_{2}$ is a probability. Subject to this constraint, we want to know whether it is possible that

$$
\left(\frac{l_{1}}{l_{2}}\right)^{2} P_{1}\left(1-\alpha_{1} P_{1}\right)+\frac{1}{\alpha_{2}}\left[\left(\frac{l_{1}}{l_{2}}\right)^{2}\left(1-\alpha_{1} P_{1}\right)+1+\epsilon\right]\left[-\epsilon-\left(\frac{l_{1}}{l_{2}}\right)^{2}\left(1-\alpha_{1} P_{1}\right)\right]>0 .
$$


Suppose that $P_{1}=1 / 2 \alpha_{1}$ (assuming $\alpha_{1}>\frac{1}{2}$ ). Then the above inequality can be cast in the form

$$
\frac{\alpha_{2}}{4 \alpha_{1}}\left(\frac{l_{1}}{l_{2}}\right)^{2}>\left(\frac{1}{2}\left(\frac{l_{1}}{l_{2}}\right)^{2}+1+\epsilon\right)\left(\epsilon+\frac{1}{2}\left(\frac{l_{1}}{l_{2}}\right)^{2}\right)
$$

which can certainly hold (e.g. if $\alpha_{2} / \alpha_{1}$ is sufficiently large), subject to the constraints that $\alpha_{1}>\frac{1}{2}$ and $\alpha_{2}>\frac{1}{2}\left(l_{1} / l_{2}\right)^{2}+1+\epsilon$, since we need $P_{2} \in[0,1]$. We have identified a parameter regime in which the mosquitoes will survive if they base their pond selection only on area, but die if they additionally try to take account of expected larval survival probability. This analysis in no way determines all sets of parameters for which this counterintuitive phenomenon happens, especially since the analysis presumes that the probabilities $P_{i}$ (defined by (5.28)) are related to the other parameters through $P_{1}=1 / 2 \alpha_{1}$ and (6.34). There is no inconsistency here - the latter two equations can certainly hold, but they will not hold generically. We must therefore emphasize that the above analysis only considers a very particular parameter regime. Other parameter regimes (which can include the requirement that $\alpha_{1} / \alpha_{2}$ be large) can be identified using other tricks. This fact is very important for a correct biological interpretation of the analysis. We will discuss this in detail in Section 8.

\section{Neumann boundary conditions}

As noted earlier, homogeneous Neumann boundary conditions are undoubtedly the most appropriate boundary conditions for small ponds that contain no predators and where the water is calm everywhere, offering a favorable habitat to larvae throughout the whole pond and not only near the edges. For Neumann boundary conditions the adult mosquitoes equation is (2.11). We consider the case where pond selection is based solely on area and the $p_{i}$ are given by $(2.7)$, and the ponds/patches (there being no distinction here) are the squares $\Omega_{i}=\left(0, l_{i}\right) \times\left(0, l_{i}\right)$. A theorem on mosquito eradication similar to Theorem 4.1 can be stated and even extracted as a particular case of that theorem by letting all the $d_{i}$ approach zero, and using $\sum_{k=0}^{\infty}(2 k+1)^{-2}=\pi^{2} / 8$. Inequality (4.26) becomes

$$
d^{\prime}(0) \sum_{i=1}^{n} l_{i}^{2}>\sum_{i=1}^{n} e^{-\mu_{i} \tau_{i}} b_{i}^{\prime}(0) l_{i}^{2}
$$

which holds if, for a particular $i, d^{\prime}(0)>e^{-\mu_{i} \tau_{i}} b_{i}^{\prime}(0)$ and $l_{i}$ is sufficiently large. We have recovered the observation, first noted in Section 4 , that an unfavorable pond that is too large will result in mosquito eradication. Although the application of homogeneous Neumann boundary conditions at the ponds edge is always entirely sensible, recall our earlier comment that the interior of a large pond may be hostile to larvae, so that the pond effectively has another interior boundary the presence of which should be (but is not in this section) recognised. Nevertheless, the presence 
of such scale effects even in the problem where homogeneous Neumann boundary conditions apply at the edges of all ponds is noteworthy because normally it is not easy to have a threshold domain size for a model with Neumann boundary conditions. In contrast to (4.26), however, it is clearly not possible to assert that (7.36) will automatically hold if all ponds are scaled down in size by the same linear factor. Note that pond information is present in (2.11) as long as it is involved in the probabilities $p_{i}$.

\section{Discussion}

We have developed a mathematical model for an insect species the adult members of which live in the air, and each juvenile in one of $n$ ponds from which it cannot escape until it reaches maturation. We have in mind mainly the mosquito and its aquatic larvae that live in ponds. Coupling between ponds is via the adults in the air. We established results on extinction and persistence of the adult mosquito population. These are rather general results that apply for $n$ ponds of general shapes and sizes, and also work regardless of the strategy adopted by the mosquitoes in selecting oviposition sites (i.e. the probabilities $p_{i}$ are kept general). We then carried out a more detailed analysis of two particular possibilities (i) the adult mosquitoes base their choice of oviposition pond purely on pond area, (ii) they additionally take account of predator density in each pond via the influence this has on larval survival probabilities. Here, we focussed on ponds represented by square patches of various sizes to facilitate a more detailed understanding of the roles of factors such as scale. If the ponds are uniformly scaled down in size below some threshold then the outcome is extinction. This is not surprising and can be compared with the dynamics of simple scalar reaction-diffusion equations on small domains under homogeneous Dirichlet boundary conditions such as the KISS model (Murray [15], Okubo and Levin [16]). More interestingly, we also showed that increasing some of the pond sizes can drive the entire mosquito population to extinction if conditions happen to be such that large ponds offer poor survival prospects. If oviposition pond selection is driven by area then mosquitoes would naturally be drawn to the large ponds, but these may turn out to be a bad choice for egg laying for one reason or another. Another patch type model with certain similarities to ours was studied by Cantrell and Cosner [8]. It was a model of a predator prey (ladybird-aphid) interaction, and under certain conditions it predicted maximum as well as minimum patch sizes for sustaining a prey population, though the ecological mechanism was different.

We identified one particular (but not the only) parameter regime in which mosquitoes will survive if they base their pond selection only on area, but die if they additionally try to take account of the probability that larvae will survive in particular ponds. To summarise, putting together the various restrictions, in order for this counterintuitive phenomenon to happen it is necessary that there 
should exist a small positive $\epsilon$ for which all of the following five restrictions hold:

$$
\begin{gathered}
b_{1}^{\prime}(0)>\frac{1}{2} d^{\prime}(0) \\
b_{2}^{\prime}(0)>\left(\frac{1}{2}\left(\frac{l_{1}}{l_{2}}\right)^{2}+1+\epsilon\right) d^{\prime}(0) \\
\frac{b_{2}^{\prime}(0)}{4 b_{1}^{\prime}(0)}\left(\frac{l_{1}}{l_{2}}\right)^{2}>\left(\frac{1}{2}\left(\frac{l_{1}}{l_{2}}\right)^{2}+1+\epsilon\right)\left(\epsilon+\frac{1}{2}\left(\frac{l_{1}}{l_{2}}\right)^{2}\right) \\
\frac{e^{-\mu_{1} \tau_{1}}}{l_{1}^{2}}\left(\frac{8 l_{1}}{\pi^{2}} \sum_{k=0}^{\infty} \frac{1}{(2 k+1)^{2}} e^{-d_{1} \pi^{2}(2 k+1)^{2} \tau_{1} / l_{1}^{2}}\right)^{2}=\frac{d^{\prime}(0)}{2 b_{1}^{\prime}(0)} \\
\frac{e^{-\mu_{2} \tau_{2}}}{l_{2}^{2}}\left(\frac{8 l_{2}}{\pi^{2}} \sum_{k=0}^{\infty} \frac{1}{(2 k+1)^{2}} e^{-d_{2} \pi^{2}(2 k+1)^{2} \tau_{2} / l_{2}^{2}}\right)^{2}=\frac{d^{\prime}(0)}{b_{2}^{\prime}(0)}\left(\frac{1}{2}\left(\frac{l_{1}}{l_{2}}\right)^{2}+1+\epsilon\right) .
\end{gathered}
$$

Now let us discuss further the biological implications of this. Firstly, recall that equations (8.40) and (8.41) arise only because of our decision to look at a particular region of parameter space. By a continuity argument, inequality (8.39) will hold for a small positive $\epsilon$ if it holds when $\epsilon=0$. Using (8.40) and (8.41) to eliminate $b_{1}^{\prime}(0)$ and $b_{2}^{\prime}(0)$, inequality (8.39) when $\epsilon=0$ can be rewritten in the form

$e^{-\mu_{1} \tau_{1}}\left(\sum_{k=0}^{\infty} \frac{1}{(2 k+1)^{2}} e^{-d_{1} \pi^{2}(2 k+1)^{2} \tau_{1} / l_{1}^{2}}\right)^{2}>e^{-\mu_{2} \tau_{2}}\left(\sum_{k=0}^{\infty} \frac{1}{(2 k+1)^{2}} e^{-d_{2} \pi^{2}(2 k+1)^{2} \tau_{2} / l_{2}^{2}}\right)^{2}$

which becomes $P_{1}>P_{2}$ where $P_{i}, i=1,2$, is the probability that a larva will successfully complete its maturation (i.e. survive all possible forms of death) in pond $i$ and is defined by (5.28). Does this imply that if $P_{1}>P_{2}$ then adult mosquitoes should base their choice of pond for oviposition on pond area alone? Here, we must recall that our analysis has focussed on a particular region of parameter space. The overall implications of the analysis should not depend on how we have labelled the ponds, and they do not. Another similar analysis can suggest that if $P_{2}>P_{1}$ then pond selection should be based on area alone. But, generically, either $P_{1}>P_{2}$ or $P_{2}>P_{1}$ must hold. Does this imply that, generically, it is always in the interests of adult mosquitoes to base their choice of pond for oviposition only on area? The answer is yes, but only if all of (8.37)-(8.41) hold, which in fact is very restrictive. It holds if the per-capita adult death rate at low densities $d^{\prime}(0)$ is relatively small, and, for the homogeneous Dirichlet problem, if $l_{1}$ and $l_{2}$ are both sufficiently small. The latter is due to the requirement that the left hand sides of (8.40) and (8.41) will both have to be small, since $d^{\prime}(0)$ is small. Those left hand sides increase with $l_{1}$ and $l_{2}$ respectively in the case of homogeneous Dirichlet boundary conditions on the patches representing both ponds. 
The biological punchline is therefore very simple in the case of two possible sites for oviposition: if the adult death rate is low, and if each pond has a hostile interior with small inhabitable zone, then it is quite useless for the adult mosquitoes to try to take account of larval survival probability in the ponds - they should base oviposition pond selection on area only.

A similar observation was made in Spencer et al. [18]. More precisely, their observation was that a strategy involving the avoidance of oviposition in ponds containing the larva-eating predator $N$. maculata might actually result in a lower equilibrium adult mosquito population (but not necessarily extinction) than an oviposition strategy that does not discriminate between ponds. However, this observation was only for very high values of their parameter for adult fecundity, which is comparable to our requirement for low values of our parameter $d^{\prime}(0)$ which measures per-capita adult mortality. At realistic parameter values, the predictions of the Spencer et al. [18] study were that C. longiareolata should always avoid ovipositing in ponds containing $N$. maculata.

Our observation that it might sometimes be to their advantage for mosquitoes to base their choice of oviposition pond on area alone, and not to try to take account of the likelihood of larvae surviving, is also perhaps only applicable for unrealistic parameter values. As noted above it includes the requirement, in the case of two square patches, that they should both be of small size. But this observation only applies if the ponds represented by these patches both have hostile interiors. If the boundary conditions are homogeneous Neumann (this case can be realised by taking $d_{1}=d_{2}=0$ ) then the left hand sides of (8.40) and (8.41) no longer involve $l_{1}$ and $l_{2}$. The case of ponds with interiors that are potentially dangerous but not completely lethal to larvae could perhaps be approximately modelled by simply reducing the values of the $d_{i}$ in the model equations below their physically correct values. This makes the smallness requirements on the $l_{i}$ more stringent and, therefore, less likely to hold.

There are a great many factors involved in the search for an oviposition site by a mosquito that are not modelled here at all. In practice it involves a complex interaction of both chemical and physical factors. Larvae can produce attractants which may lead to a preference for ponds that previously held conspecific larvae. These substances can remain active for long periods. Larval density can be important, with some species preferring uncrowded conditions. Physical aspects other than area, such as pond brightness, are known to be important for some species. Also, as noted earlier, the time available for the oviposition pond search may be restricted (it varies with species) and so it may not be possible to visit all potential ponds. For an extensive discussion of these points and others, see Bentley and Day [3] and the references therein.

Acknowledgement. We thank the three referees for their comments that have led to a greatly improved paper. 


\section{References}

[1] R. Barrera. Competition and resistance to starvation in larvae of containerinhabiting Aedes mosquitoes. Ecol. Entomol. 21 (1996), 117-127.

[2] S. Bédhomme, P. Agnew, C. Sidobre \& Y. Michalakis, Pollution by conspecifics as a component of intraspecific competition among Aedes aegypti larvae. Ecol. Entomol. 30 (2005), 1-7.

[3] M.D. Bentley and J.F. Day, Chemical ecology and behavioral aspects of mosquito oviposition. Ann. Rev. Entomol. 34 (1989), 401-421.

[4] L. Blaustein, M. Kiflawi, A. Eitam, M. Mangel and J.E. Cohen, Oviposition habitat selection in response to risk of predation in temporary pools: mode of detection and consistency across experimental venue. Oecologia 138 (2004), 300-305.

[5] L. Blaustein and B.P. Kotler, Oviposition habitat selection by the mosquito Culiseta longiareolata: effects of conspecifics, food, and green toad tadpoles. Ecol. Entomol. 18 (1993), 104-108.

[6] J.S. Edgerly, M. Mcfarland, P. Morgan and T. Livdahl, A seasonal shift in egg-laying behaviour in response to cues of future competition in a treehole mosquito. J. Animal Ecol. 67 (1998), 805-818.

[7] J.G. Bond, J.I. Arredondo-Jiménez, M.H. Rodríguez, H. Quiroz-Martínez, T. Williams, Oviposition habitat selection for a predator refuge and food source in a mosquito. Ecol. Entomol. 30 (2005), 255-263.

[8] R.S. Cantrell and C. Cosner, Models for predator-prey systems at multiple scales. SIAM Rev. 38 (1996), 256-286.

[9] A. Eitam and L. Blaustein, Oviposition habitat selection by mosquitoes in response to predator (Notonecta maculata) density. Physiol. Entomol. 29 (2004), 188-191.

[10] W. Gu, J.L. Regens, J.C. Beier, and R.J. Novak, Source reduction of mosquito larval habitats has unexpected consequences on malaria transmission. Proc. Natl. Acad. Sci. USA 103 (2006), 17560-17563.

[11] W. Gu, J. Utzinger and R.J. Novak, Habitat-based larval interventions: a new perspective for malaria control. Am. J. Trop. Med. Hyg. 78 (2008), 2-6.

[12] J.K. Hale and P. Waltman, Persistence in infinite-dimensional systems. SIAM J. Math. Anal. 20 (1989), 388-395.

[13] S.B. Heard, Imperfect oviposition decisions by the pitcher plant mosquito (Wyeomyia smithii). Evol. Ecol. 8 (1994), 493-502. 
[14] C.J. Koenraadt \& W. Takken, Cannibalism and predation among larvae of the Anopheles gambiae complex. Med. Vet. Entomol. 17 (2003), 61-66.

[15] J.D. Murray, Mathematical Biology. Vol. II. Spatial models and biomedical applications. Third edition. Interdisciplinary Applied Mathematics, 18. Springer-Verlag, New York, 2003.

[16] A. Okubo and S.A. Levin, Diffusion and Ecological Problems: Modern Perspectives. Second edition. Interdisciplinary Applied Mathematics, 14. Springer-Verlag, New York, 2001.

[17] H.L. Smith, Monotone Dynamical Systems: An Introduction to the Theory of Competitive and Cooperative Systems. Mathematical Surveys and Monographs, 41. American Mathematical Society, Providence, RI, 1995.

[18] M. Spencer, L. Blaustein and J.E. Cohen. Oviposition habitat selection by mosquitoes (culiseta longiareolata) and consequences for population size. Ecology 83 (2002), 669-679.

[19] H.R. Thieme, Convergence results and a Poincaré-Bendixson trichotomy for asymptotically autonomous differential equations. J. Math. Biol. 30 (1992), 755-763.

[20] WHO, Vector Control for Malaria and Other Mosquito Borne Diseases. WHO Technical Report Series, Geneva, 1995. 